\title{
DETERMINACIÓN Y UBICACIÓN GEO-REFERENCIAL DE LAS PEQUEÑAS CENTRALES HIDROELÉCTRICAS EXISTENTES EN LA REPÚBLICA DOMINICANA
}

\author{
Georeferencing determination and location of small hydroelectric \\ power plants in the Dominican Republic
}

\author{
MARTÍN MELÉNDEZ ${ }^{1}$
}

Cómo citar: Meléndez, M. (2018). Determinación y ubicación geo-referencial de las pequeñas centrales hidroeléctricas existentes en la República Dominicana. Ciencia, Ingenierías y Aplicaciones, 1(1), 61-70. doi:http://dx.doi.org/10.22206/cyap.2018.v1i1.pp61-70

\begin{abstract}
Resumen
En esta nota se discute la ubicación geo-referencial de pequeñas centrales hidroeléctricas de la República Dominicana. El objetivo es definir una base de datos a través de la cual se pueda dar seguimiento de manera rápida y eficiente al funcionamiento de esta fuente de energía eléctrica. Las pequeñas centrales hidroeléctricas permiten suministrar energía eléctrica a comunidades aisladas a las cuales no sería económicamente factible suministrar energía tradicional, reduciendo la carga y el costo del sistema eléctrico nacional.
\end{abstract}

Palabras clave: pequeñas hidroeléctricas; georreferenciación; energía hidroeléctrica; energía eléctrica.

\begin{abstract}
This note discusses the georeferencing location of small hydroelectric power plants in the Dominican Republic. The objective is to develop a database through $w$ hich we can assess the performance of this source of electric energy. Small hydroelectric power plants allow to provide electric energy to small or isolated communities to which it would be uneconomical or unfeasible to directly connect to the national power grid.
\end{abstract}

Keywords: Small hydro power plants; georeferencing; hydroelectricity; electric energy.

\footnotetext{
1 Instituto Tecnológico de Santo Domingo (INTEC), República Dominicana. Correo electrónico: martin. melendez@intec.edu.do
} 


\section{Introducción}

El sector energético en la República Dominicana dispone de un gran potencial de cobertura eléctrica que, de acuerdo con el último censo de la República Dominicana (2010), la tasa de electrificación urbana era de un 92.3, mientras que la cobertura eléctrica en las áreas rurales llegaba apenas a un $76.6 \%$, lo cual se traduce en unas 350,000 viviendas rurales sin electricidad. Esta baja cobertura energética en las zonas rurales tiene varias razones (Gobierno Dominicano, 2012), entre ellas cabe mencionar:

- La baja densidad poblacional

- Las grandes distancias de las redes de distribución

- El difícil acceso a las comunidades

- Los elevados costos de infraestructura de distribución

- El bajo poder adquisitivo de la población.

En países como República Dominicana existe una necesidad creciente del suministro de energía a todos los niveles. Con el crecimiento descontrolado que tiene la población y la necesidad de energía eléctrica de las personas para satisfacer sus necesidades laborales y familiares, se hace cada vez más difícil abastecer a más comunidades de energía eléctrica y esto se ha convertido en uno de los principales problemas en los países en vía de desarrollo (CEPAL, 2010).

Hasta ahora, la producción energética en la República Dominicana depende, en su mayoría, de los combustibles fósiles y se caracteriza por los altos costos de energía para los consumidores finales -industria, comercio, turismo y habitantes-(Gobierno Dominicano, 2010). La inestabilidad de los precios del petróleo y otros combustibles, sumado al aumento de la demanda energética, ha dado lugar a constantes aumentos de precio, que se reflejan en todos los ámbitos de la sociedad dominicana (Monegro, 2005). 
La República Dominicana está considerada como un país con gran potencial hidroeléctrico, con un índice de precipitaciones promedio que oscila entre 1400 y $1800 \mathrm{~mm}$, lo que indica un alto contenido de recursos hídricos en la zona que, combinado con la orografía, da paso a un buen aprovechamiento de las masas de agua y su potencial hidráulico presenta posibilidades de aprovechamiento de pequeños saltos hidráulicos para generar electricidad (Gobierno Dominicano, 2015). Esta producción de energía eléctrica en las zonas de montaña, aisladas de las redes existentes, puede reducir costos de expansión de estas, a la vez que lleva esta preciada energía a sitios remotos.

El uso hidroeléctrico del agua es no consuntivo, es decir, no consume nada de agua. El agua después de pasar por los conductos y las maquinarias se devuelve al cauce de forma íntegra e invariable en cuanto a sus condiciones físicas, químicas y biológicas. Este uso lo único que consume es energía, no agua. Por esto podemos considerar las centrales hidroeléctricas como fuentes de energía renovable, ya que con solo agregarle energía al agua ya estamos en condiciones de producir de nuevo electricidad con la misma agua.

Este hecho permite depender menos de las fuentes de combustibles fósiles, muy costosos y con importantes emisiones de $\mathrm{CO}_{2}$. $\mathrm{Si}$ establecemos un equilibrio entre las necesidades económicas y la consciencia ecológica, la energía hidroeléctrica destaca en términos de rentabilidad, sostenibilidad y disponibilidad (De Miguel \& Tavares, 2012).

Una Pequeña Central Hidroeléctrica $(\mathrm{PCH})$ es una tecnología de energía renovable a pequeña escala, bien desarrollada, que puede contribuir a la mejora del acceso a la electricidad en las zonas rurales y que se convierte en parte de la solución para el desarrollo de los seres humanos y los pueblos beneficiados (http://www.fao.org/ 
Noticias/2000/001003-s.htm). Gustavo Best, entrevistado por Enrique Yeves, Servicio de Radio y Televisión de la FAO, sobre energía solar en las zonas rurales. La energía hidroeléctrica es la mayor fuente de energía limpia y renovable, debido a esto ha aumentado su uso en función de generar un impacto en el desarrollo socioeconómico y lograr que sus efectos puedan palear las consecuencias del calentamiento global. Recibir beneficios directos de la utilización de los recursos hidráulicos en comunidades de montaña puede ser clave para integrar a los pobladores en la preservación de los recursos hídricos.

Proyectar, construir y poner en marcha una central hidroeléctrica no es tarea fácil. Para hacerlo hay que tomar en consideración múltiples aspectos del problema, desde la elección del sitio adecuado hasta la explotación del aprovechamiento. Todo esto exige un amplio espectro de conocimientos sobre ingeniería y financiación, además de que precisa establecer relaciones con las autoridades locales de gobierno, la sociedad civil y las autoridades nacionales.

Es responsabilidad de nuestras autoridades suministrar dicha energía, pero para encarar los altos costos de extensión de las redes eléctricas y sus concebidas pérdidas de energía se hace necesario buscar alternativas viables, como sería una red de pequeñas hidroeléctricas, diseñadas y construidas en diferentes puntos de una o varias cuencas hidrográficas.

En un estudio realizado por un equipo de técnicos del Instituto Tecnológico de Santo Domingo (INTEC) para el Ministerio de Energía y Mina, financiado por el Banco Interamericano de Desarrollo, se afirma que en la República Dominicana existen en la actualidad más de $64 \mathrm{PCH}$, además de 33 presas convencionales dedicadas a la producción eléctrica, alcanzando cerca de $180 \mathrm{MW}$. 
Para el levantamiento de la data existente en el país se realizó una investigación exploratoria y descriptiva de los datos cualitativos y cuantitativos. Las instituciones consultadas para este trabajo fueron:

- Instituto Dominicano de Recursos Hidráulicos (INDRHI)

- Empresa de Generación Hidroeléctrica Dominicana (EGEHID)

- Comisión Nacional de Energía (CNE)

- Unidad de Electrificación Rural y Sub-urbana (UERS)

- Programa de Pequeños Subsidios del Programa de Naciones Unidas para el Desarrollo (PNUD-PPS)

- National Rural Electric Cooperative Association (NRECA)

De igual manera, se trabajó con las estaciones hidrometeorológicas de la Oficina Nacional de Meteorología (ONAMET).

Además de la información recabada en estas instituciones, el proyecto incorporó al estudio un análisis hidrológico de cada punto, que contiene los parámetros físicos de la cuenca, así como un análisis de las precipitaciones.

También, se añadieron parámetros hidráulicos, cálculos de escorrentías, cálculos estimados de los embalses, tiempo de descarga de estiaje, obras hidráulicas necesarias y potencia de la turbina. Para cada punto se realizó un análisis geológico y geotécnico. Se puede ver la base de datos en su estado actual en el enlace electrónico https://sites.google.com/view/proyectopch/home.

De todas estas minicentrales hidroeléctricas identificadas en su estado actual el balance es el siguiente: 64 en operación (60\%), 28 en estudio (26\%), 14 en construcción (13\%), y una está fuera de servicio (1\%); para un total de $107 \mathrm{PCH}$. La figura 1 gráfica lo antes detallado. 
Figura 1. Estado actual de las pequeñas centrales hidroeléctricas de la República Dominicana

\section{Estado de las PCHs}

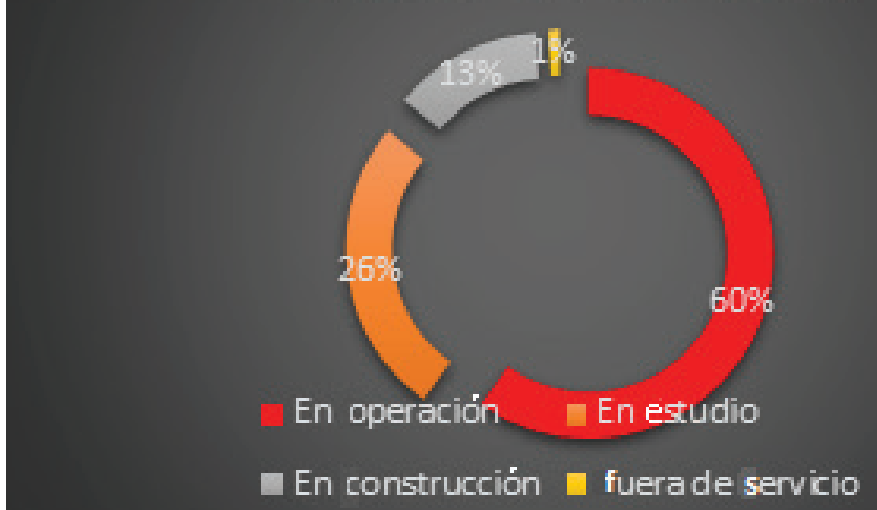

En la siguiente tabla de presentan los datos que se relacionan con la inversión que se realizó.

Tabla 1. Datos relacionados con la inversión realizada

Producción teórica

Familias beneficiadas

Caudal utilizado

Áreas de las cuencas utilizadas

Generación eléctrica

Costo total

Costo promedio por $\mathrm{PCH}$

Costo por KW

Costo por GWhora/anual

Costo por familia
180.92 MW

$$
\begin{array}{r}
6,810 \\
2,456 \mathrm{~m}^{3} / \mathrm{s} \\
5,415 \mathrm{~km}^{2} \\
575,737.29 \mathrm{GW} / \mathrm{año} \\
\mathrm{RD} \$ 8,057,147,121.85 \\
\mathrm{RD} \$ 75,300,440.38 \\
\mathrm{US} \$ 1,583,938 \\
\mathrm{RD} \$ 44,761,699 \\
\mathrm{US} \$ 941,558 \\
\mathrm{RD} \$ 13,994.49 \\
\mathrm{RD} \$ 1,183,134.67
\end{array}
$$

Fuente: Elaboración propia. Basada en los datos obtenidos en nuestra investigación y que están plasmados en la base de datos que se puede ver en: https://sites.google.com/view/proyectopch/home; realizada por el proyecto en seis meses de investigación.

En la figura que veremos a continuación se presenta la potencia bruta de las centrales hidroeléctricas de nuestro país. 
Figura 2. Potencia bruta de las pequeñas centrales hidroeléctricas de la República Dominicana

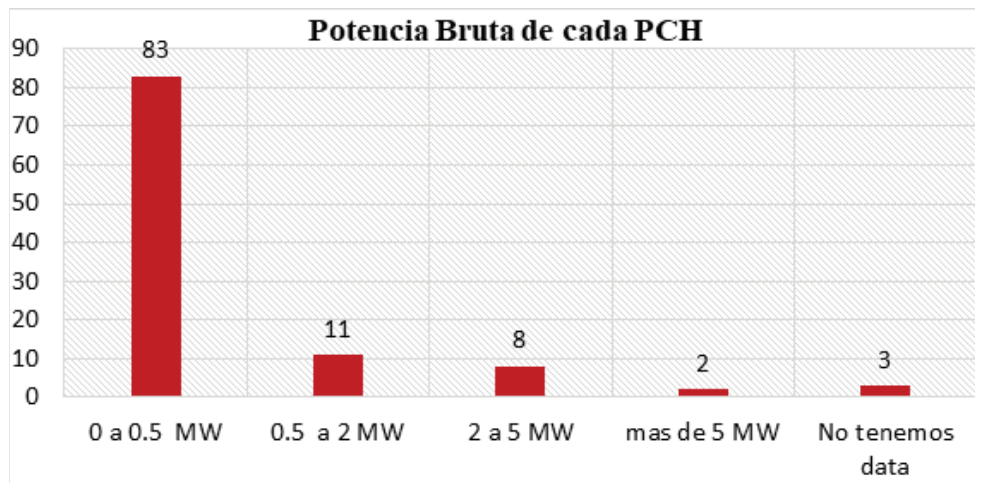

Fuente: Elaboración propia. Basada en los datos obtenidos en nuestra investigación y que están plasmados en la base de datos que se puede ver en: https://sites.google.com/view/proyectopch/home; realizada por el proyecto en seis meses de investigación.

Como parte del proyecto se determinaron los parámetros físicos de las cuencas donde se encuentran las PCH y se realizó un análisis hidrológico de cada una de las subcuencas. Estos parámetros son el área de la cuenca, la pendiente del cauce principal, la densidad de drenaje, la longitud del cauce principal y las longitudes de los afluentes.

Con los datos suministrados por la ONAMET, se determinaron los caudales promedios, máximos y de estiaje que podía tener cada cuenca, con un período de retorno de 50 años (ver figura 3 ).

Figura 3. Caudal promedio para un período de retorno de 50 años

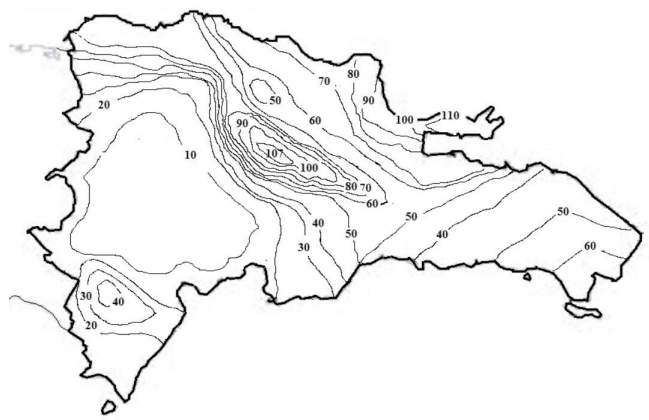

Basado en los datos meteorológicos suministrados por la Oficina Nacional de Meteorología, para los últimos treinta años, y analizados y trabajado por el equipo del proyecto, para obtener los datos presentados y necesarios para el proyecto. 
Si tomásemos las 10 regiones hidrográficas principales del país, podríamos ubicar las 107 pequeñas centrales hidroeléctricas $(\mathrm{PCH})$ diseminadas por toda el área de la República Dominicana de la siguiente manera: En el río Yaque del Norte se encuentran 39 PCHs, para un 36\%; en la región norte tenemos 13 PCHs entre los ríos Yásica, Bajabonico, Nagua y Boba, para un 13\%; la región de Valdesia, con los ríos Ocoa, Nizao y Nizaíto poseen 17 PCHs, para un 16\%; el río Yaque del Sur, con un 10\%; el río Yuna con el 7\%, con 8 PCHs; y el río Artibonito con $7 \mathrm{PCH}$, para un 7\%; el río Soco y el río Ozama con 2 PCHs cada uno, para un 4\%. El otro 7\% corresponde a PCHs en la Sierra de Bahoruco, Dajabón y La Hoya del Lago Enriquillo (ver figura 4).

Figura 4. Cuencas hidrográficas de la República Dominicana

Cuencas Hidrográficas

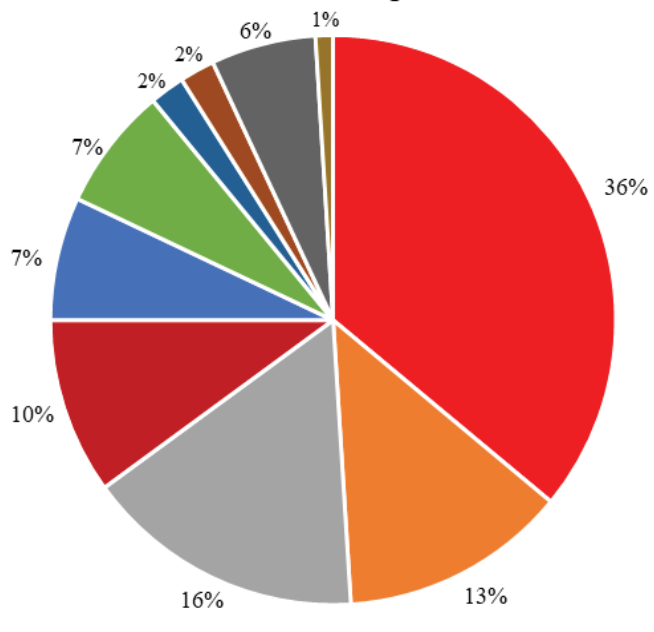

- Yaque del Norte
- Region Valdesia (Ocoa, Nizao y Nizaito)
- Rio Yuna
- Rio Soco
- Hoya Lago Enriquillo

- Region norte (Yasica, Bajabonico, Nagua y Boba)

- Yaque del Sur

- Rio Artibonito

- Rio Ozama

- Otros

Fuente: elaboración propia. Basada en los datos obtenidos en nuestra investigación y que están plasmados en la base de datos que se puede ver en: https://sites.google.com/view/proyectopch/home; realizada por el proyecto en seis meses de investigación. 
Una vez determinada la base de datos en un sistema de información geográfico georreferenciado los saltos hidráulicos que recogen los estudios existentes podemos recomendar lo siguiente:

Concluido el estudio de todos los puntos donde existen o se han realizado estudios para $\mathrm{PCH}^{\prime}$ s, se construyó una base con los datos de cada uno de ellos, incluyendo una georreferenciación de los mismos, los cuales se llevaron a un mapa GIF; analizando los resultados del mismo, podemos hacer las siguientes recomendaciones:

Esta base de datos debe servir de incentivo para auspiciar el uso de pequeñas centrales hidroeléctricas en el país.

1. Mantener actualizada la base de datos con los proyectos que se puedan ir estudiando y/o ejecutando en un futuro.

2. Se sugiere la formación de un observatorio de los recursos hídricos a nivel nacional.

3. Como seguimiento a este proyecto, se hace imprescindible un estudio pormenorizado de cada una de las cuencas, subcuencas y microcuencas del país, investigando el potencial hídrico de cada una de ellas, empezando por la zona fronteriza.

\section{Referencias}

CEPAL. (2010). Objetivos de Desarrollo del Milenio, Avances en la Sostenibilidad Ambiental del Desarrollo en América Latina y El Caribe. United Nations, CEPAL.

De Miguel, C. \& Tavares, M. (2012). El desafio de la sostenibilidad ambiental en América Latina y el Caribe. Santiago de Chile: CEPAL: textos seleccionados 2012-2014.

Gobierno Dominicano. (2010). Prospectiva de la Demanda de Energía de República Dominicana 2010- 2030. Santo Domingo: Comisión Nacional de Energía. 
Gobierno Dominicano. (2012). IX Censo Nacional de Población y Vivienda 2010. Informe general, Volumen 1, Santo Domingo. Gobierno Dominicano. (2015). Potencial Hidráulico para Pequeñas, Mini, y Micro-Hidroeléctricas en la República Dominicana. Santo Domingo: Comisión Nacional de Energía, gerencia de Fuentes Alternas y Uso racional de la Energía.

Monegro, J. (2005). Secretariado técnico de la Presidencia, Crecimiento de los Precios del Petróleo y sus Repercusiones en la Economía Dominicana. 